\title{
Does the PKU diet contribute to impaired renal function?
}

\author{
Denise M. Ney
}

Received: 15 February 2013 /Revised: 25 March 2013 / Accepted: 19 April 2013 /Published online: 8 May 2013

(C) SSIEM and Springer Science+Business Media Dordrecht 2013

The recent report by Hennermann et al provides novel evidence of impaired renal function, proteinuria and arterial hypertension in adolescent and adult PKU patients (median age 24 years) treated lifelong with the low-phe, amino acid (AA)-based diet (Hennermann et al 2012). I wish to call attention to our recent research publications (not cited by the authors) that support their findings and highlight the need for additional research to elucidate the contribution of the PKU diet to impaired renal function.

Briefly, impaired renal function was observed in $19 \%$ of subjects (11 out of 59) based on a glomerular filtration rate (GFR, measured by ${ }^{51} \mathrm{Cr}$-EDTA) that was below the normal range and associated with longer lifelong years of diet time and greater proteinuria compared to subjects with a normal GFR (Supplementary Table 2, Hennermann et al 2012). Lifelong plasma phe concentration did not correlate with GFR and total dietary protein intake was within the range of a typical Western diet $(0.75-1.14 \mathrm{~g}$ pro/kg/day). Given that the majority of nitrogen is provided by a mixture of AAs rather than intact dietary protein in the PKU diet, evidence presented by Hennermann et al suggests that the low-phe AA diet may contribute to alterations in renal function that warrant prospective, controlled investigation to identify the underlying pathophysiology.

Our recent studies comparing low-phe diets comprised of AAs versus primarily intact protein from glycomacropeptide (GMP) in murine and human PKU are consistent with the

Communicated by Eva Morava

\section{M. Ney $(\square)$}

Department of Nutritional Sciences,

University of Wisconsin-Madison, 1415 Linden Drive,

Madison, WI 53706-1571, USA

e-mail: ney@nutrisci.wisc.edu potential for the AA-based PKU diet to impair renal function. Specifically, chronic ingestion of the AA diet significantly increased renal mass, water intake and urine output (Solverson et al 2012a) and also significantly reduced bone size and strength (Solverson et al 2012b) in both $\mathrm{Pah}^{\text {enu2/enu2 }}$ and wild type littermates compared with the GMP diet. PKU subjects fed the AA diet in an inpatient metabolic study showed significantly lower plasma AA concentrations, higher blood urea nitrogen concentrations, and lower carbon dioxide at $2.5 \mathrm{~h}$ after a meal compared with the GMP diet (van Calcar et al 2009). These changes suggest that the AA diet provides a high dietary acid load and invokes greater renal workload, due to rapid absorption of AAs followed by subsequent degradation to urea, compared with the low-phe GMP diet.

The low-phe, AA-based diet implemented in infancy has shown tremendous success in preventing severe cognitive impairment due to PKU. Unfortunately, there is evidence of suboptimal health outcomes in PKU subjects treated with the AA diet including neurocognitive/psychosocial issues, oxidative stress, skeletal fragility (Enns et al 2010), and now, the potential for impaired renal function. In order to develop improved treatments that minimize these chronic complications associated with PKU, additional research with controlled dietary treatments is needed.

Conflict of interest The research cited was supported by grants awarded to D. M. Ney from the National Institutes of Health, National PKU Alliance, and United States Department of Agriculture HATCH grant WISO1517. D. M. Ney is a co-inventor on U.S. Patent Application US-2010-0317597, entitled "Glycomacropeptide (GMP) medical foods for nutritional management of phenylketonuria (PKU) and other metabolic disorders," which is held by the Wisconsin Alumni Research Foundation and licensed to Cambrooke Foods, LLC. A percentage of all royalty payments is awarded to the inventors. 


\section{References}

Enns GM, Koch R, Brumm V et al (2010) Suboptimal outcomes in patients with PKU treated early with diet alone: revisiting the evidence. Mol Genet Metab 101:99-109

Hennermann JB, Roloff S, Gellermann J et al (2012) Chronic kidney disease in adolescent and adult patients with phenylketonuria. $\mathrm{J}$ Inherit Metab Dis 2012 Nov 09 [epub ahead of print]

Solverson P, Murali SG, Brinkman AS et al (2012a) Glycomacropeptide, a low-phenylalanine protein isolated from cheese whey, supports growth and attenuates metabolic stress in the murine model of phenylketonuria. Am J Physiol Endocrinol Metab 302:E885E895

Solverson P, Murali SG, Litscher SJ, Blank RD, Ney DM (2012b) Low bone strength is a manifestation of phenylketonuria in mice and is attenuated by a glycomacropeptide diet. PLoS One 7:e45165

Van Calcar SC, Macleod EL, Gleason ST et al (2009) Improved nutritional management of phenylketonuria by using a diet containing glycomacropeptide compared with amino acids. Am J Clin Nutr 89:1068-1077 